Supporting Information for:

\title{
Fast magic angle spinning solid state 1H NMR reveals structural relationships in the high explosive 2,6-diamino-3,5-dinitropyrazine-1-oxide (LLM-105)
}

\author{
Harris E. Mason ${ }^{1}$, Ginger J. Guillen ${ }^{1}$, Alexander E. Gash ${ }^{1}$
}

${ }^{1}$ Physical and Life Sciences Directorate, Lawrence Livermore National Laboratory, 7000 East Ave, Livermore CA 94550

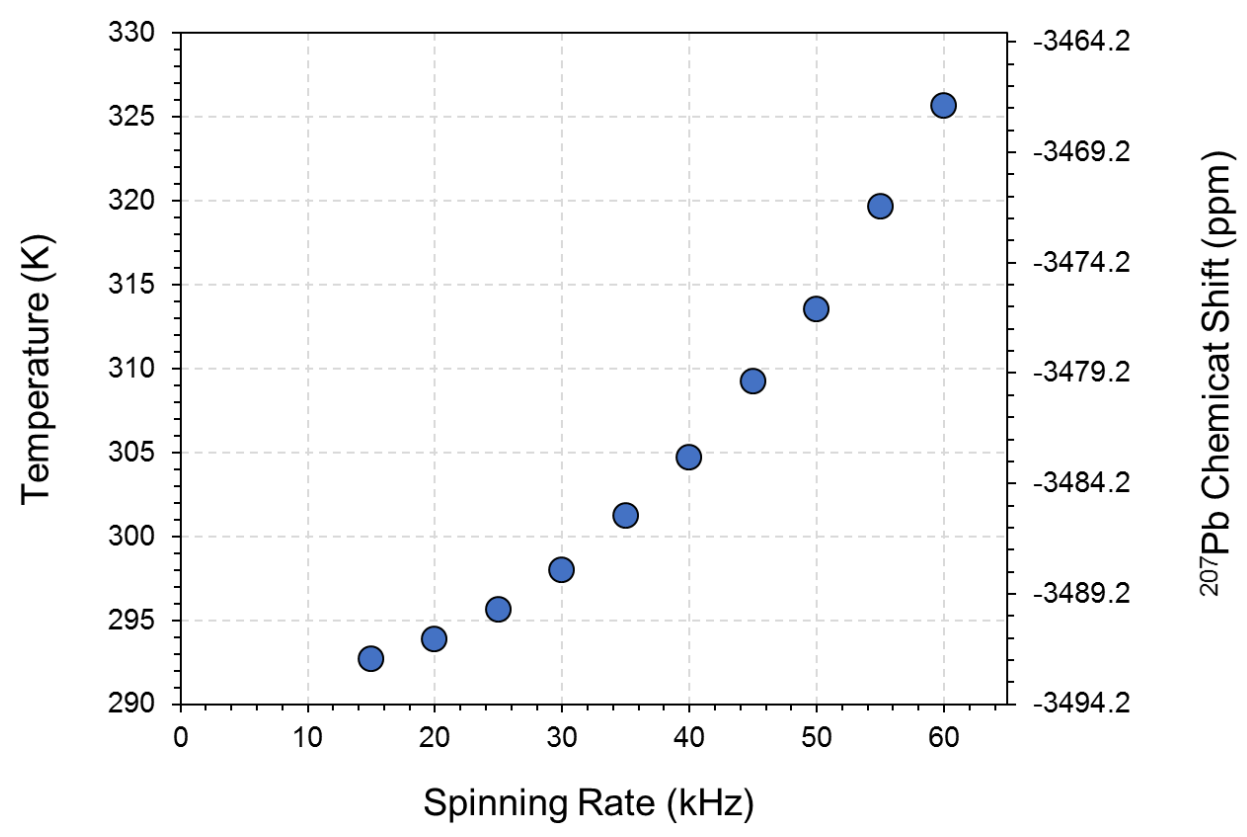

Figure S1. Sample temperature and corresponding chemical shift of $\mathrm{Pb}\left(\mathrm{NO}_{3}\right)_{2}$ in the $1.3 \mathrm{~mm}$ rotor plotted as a function of the sample spinning speed. 


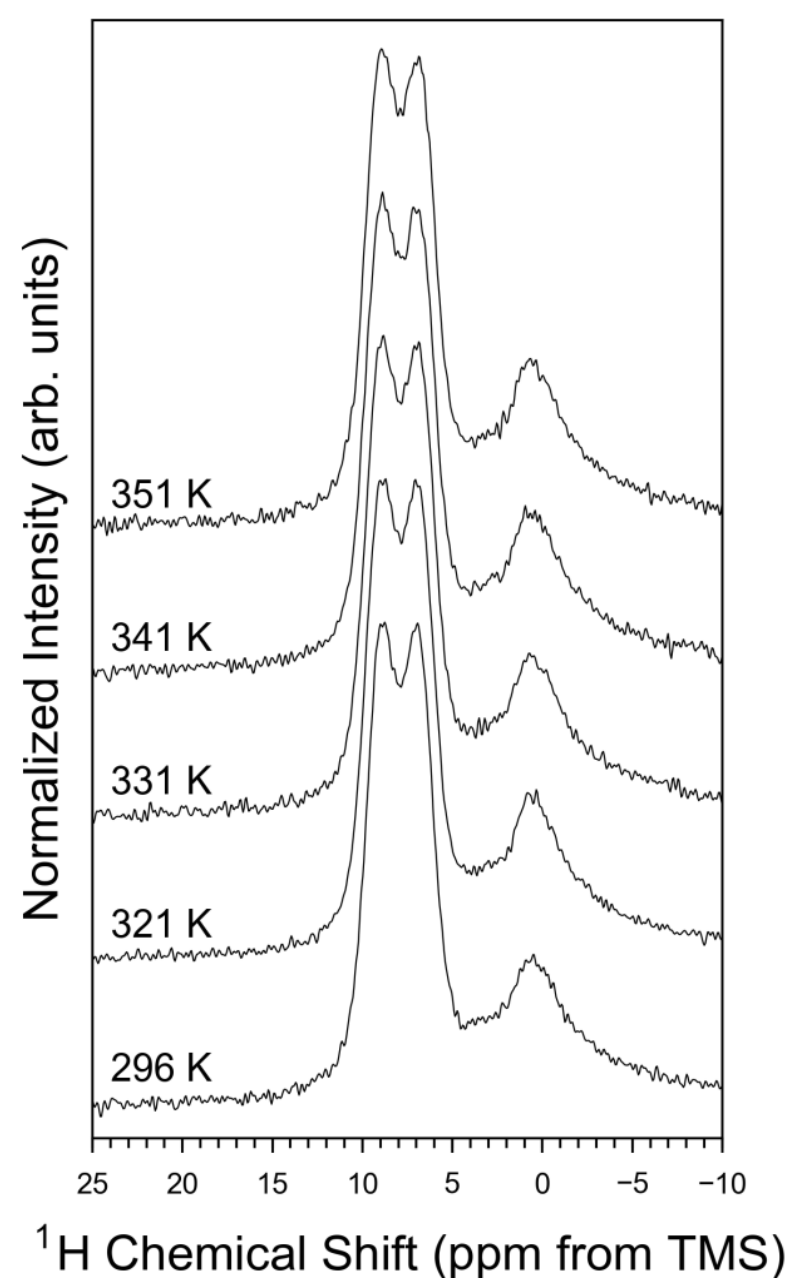

Figure S2. ${ }^{1} \mathrm{H}$ SP/MAS NMR spectra collected of LLM-105 at $50 \mathrm{kHz}$ spinning speed and the indicated temperature. A $30 \mathrm{~s}$ recycle delay was used to collect the data. 

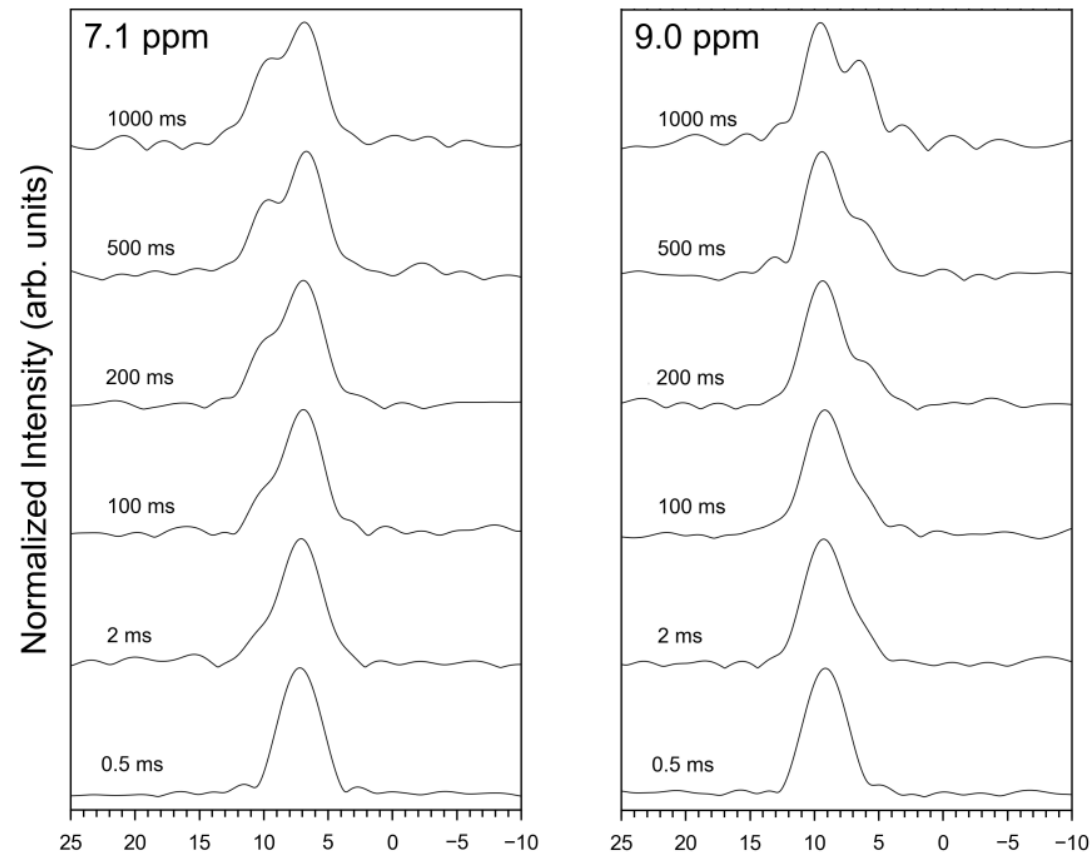

${ }^{1} \mathrm{H}$ Chemical Shift (ppm from TMS) ${ }^{1} \mathrm{H}$ Chemical Shift (ppm from TMS)

Figure S3. Indirect dimension (F1) slices taken at given positions from the ${ }^{1} \mathrm{H}$ EXSY NMR spectra collected of LLM-105 at the indicated mixing times. 


\section{Pulse sequence for Bruker Avance III spectrometer running Topspin 3.x}

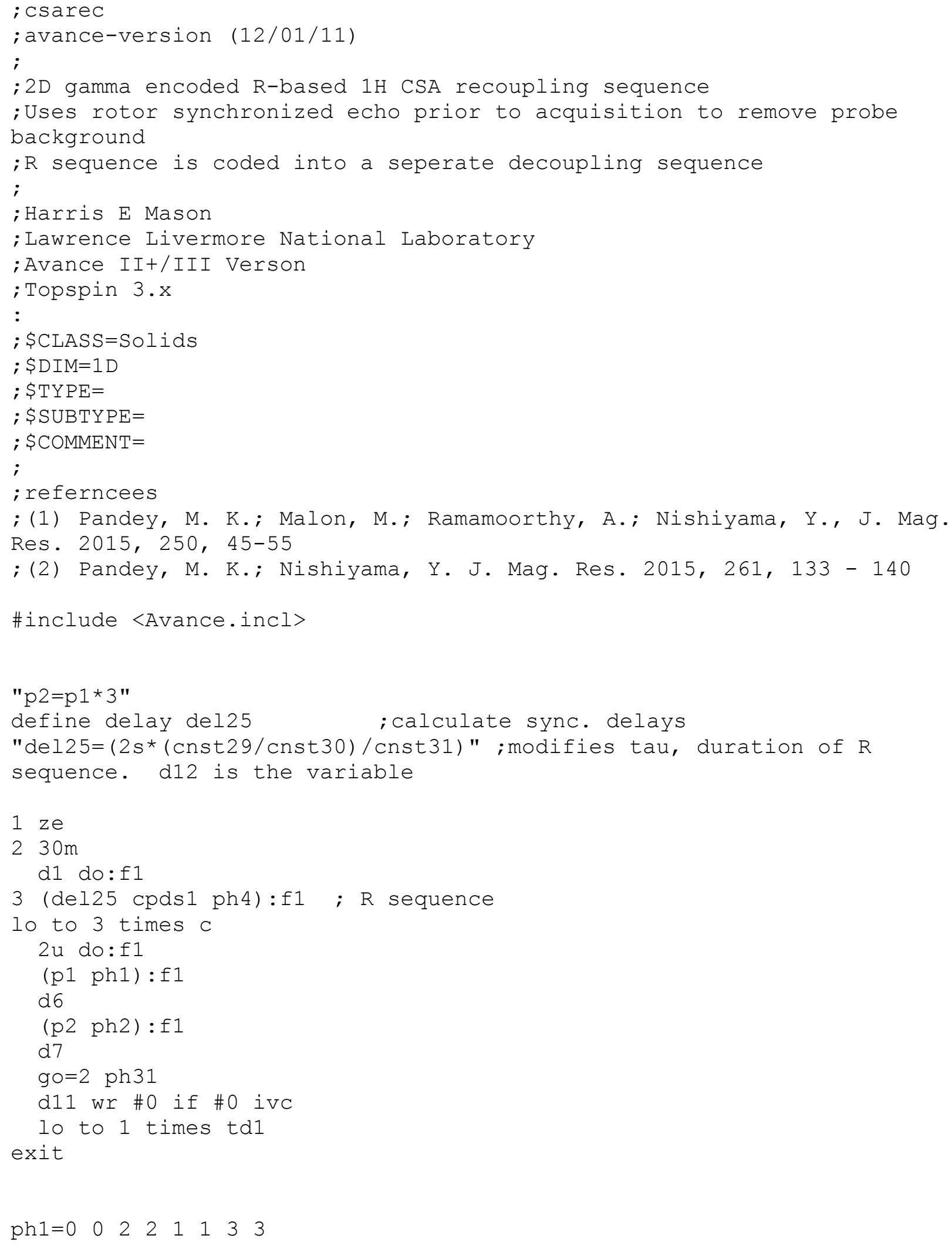




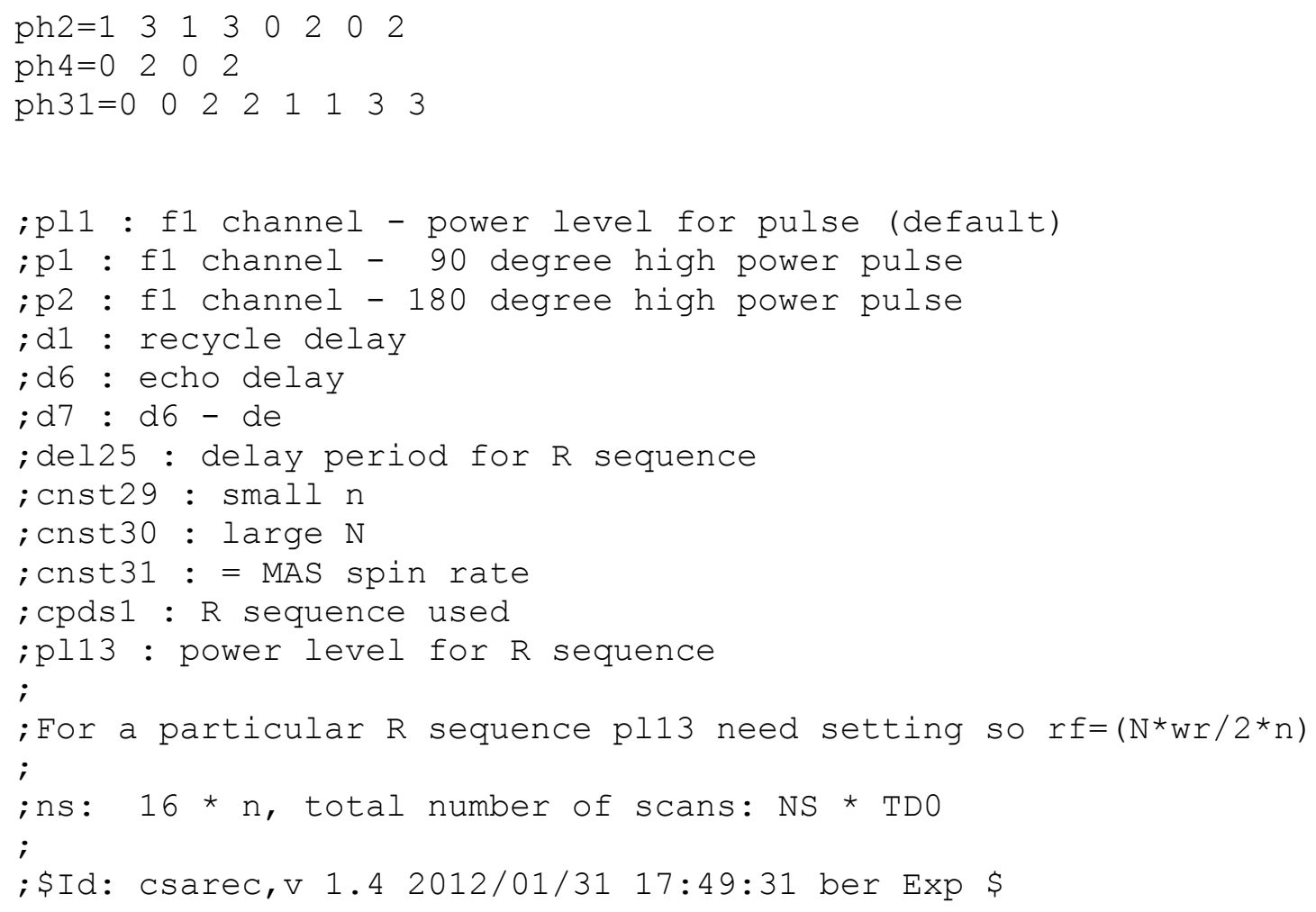


CPD Program for the $R 125^{4}$ sequence

$0.5 \mathrm{u} \mathrm{pl}=\mathrm{pl} 12$

1 p31:60

p32:240

p31:300

p32:120

jump to 1 